\title{
A pesquisa sobre envelhecimento humano no Brasil: grupos e linhas de pesquisa
}

\author{
The research about human aging in Brazil: \\ groups and research lines
}

Shirley Donizete Prado 1 Jane Dutra Sayd 1

\footnotetext{
1 Universidade do Estado do Rio de Janeiro (UERJ), Universidade Aberta da Terceira Idade (UnATI), Gerência de Pesquisa Centro de Referência e Documentação sobre Envelhecimento (CRDE). Rua São Francisco Xavier 524/10o andar, Bloco F, Pavilhão João Lyra Filho, Maracanã, 20559-900, Rio de Janeiro RJ. pradosd@uerj.br
}

Abstract We describe the scientific research about human aging in Brazil from the 4.1 version of the Executive Committee of the Research Groups in Brazil (2002) of the Scientifical and Technological Development National Council ( $\mathrm{CNPq})$. The criterion to identify the studied groups was the one of, at least, the development of a research line related to the human aging, and the search in the data base was done from key words related to the human aging. There were one hundred and fortyfour groups identified, two hundred and nine research lines, and five hundred and eleven researchers. We discussed the fields of knowledge in which these groups are enclosed, the period in which they apeeared, and their geographical and institutional distribution.

Key words Aging, Gerontology, Research groups, Research lines, Brazil
Resumo Descrevemos a pesquisa científica sobre envelhecimento humano no Brasil a partir da versão 4.1 do Diretório dos Grupos de Pesquisa no Brasil (2000) do Conselho Nacional de Desenvolvimento Científico e Tecnológico (CNPq). O critério para a identificação dos grupos estudados foi o desenvolvimento de, pelo menos, uma linha de pesquisa referente ao envelhecimento humano, e a busca na base de dados foi realizada a partir de palavras-chave associadas ao envelhecimento humano. Foram identificados 144 grupos, 209 linhas de pesquisa e 511 pesquisadores. Discutimos as áreas de conhecimento em que se inserem estes grupos, o período de seu surgimento e sua distribuição geográfica e institucional.

Palavras-chave Envelhecimento, Gerontologia, Grupos de pesquisa, Linhas de pesquisa, Brasil 


\section{A “descoberta da velhice" e a produção científica relativa ao envelhecimento humano no Brasil}

Os idosos, a velhice e o processo de envelhecimento humano vêm ganhando espaços cada vez maiores no cenário nacional ao longo da segunda metade do século 20 , alcançando um verdadeiro boom gerontológico em sua última década. Neri e Debert (1999) nos falam sobre esses acontecimentos: Esse interesse se traduziu na constituição de centros de estudo e de formação de profissionais voltados para trabalhar com idosos, na abertura de espaços para reuniões e discussões sobre essa temática e na criação de áreas especificamente voltadas para o estudo do envelhecimento, no interior de disciplinas acadêmicas plenamente constituídas.

Em afinidade com esta perspectiva de crescimento do interesse pelas questões atinentes ao envelhecimento humano, Groisman (2002) observa que: $O$ Brasil parece ter definitivamente "descoberto" a velhice. Depois do Ano Nacional do Idoso, em 1999, ganhou destaque o mais recente congresso da Sociedade Brasileira de Geriatria e Gerontologia ( $S B G G$ ), realizado na capital do país, em junho de 2000. O título, interessante, mas sem uma correspondência real com a programação oferecida, parecia inscrever-se nesse "modismo" do novo milênio: Século 21 - Envelhecimento, Tecnologia e Ética.

Ano após ano, um número cada vez maior de participantes inscreve-se no evento. O Congresso Brasileiro de Geriatria e Gerontologia parece simbolizar a importância que o envelhecimento ganhou em nossa sociedade. Convertida em matéria de interesse público, a velhice vem sendo cada vez mais tematizada pela mídia, que abre espaço para um crescente número de especialistas e de serviços voltados para essa "faixa etária". A "terceira idade" torna-se uma espécie de moda, com a constituição de um mercado de consumo específico. No centro de todo esse movimento, destaca-se a própria gerontologia, como portavoz oficial dos novos discursos sobre a velhice (Groisman, 2002).

Nesse contexto, é possível considerar que a produção de conhecimento sobre envelhecimento também venha apresentando incrementos importantes. Entretanto, poucas são as publicações que nos trazem informação de abrangência nacional acerca das atividades desenvolvidas nas instituições de ensino e pesquisa, nos serviços de saúde, ou em outros espaços, muitos dos quais ainda por serem identificados.
No que se refere à pesquisa científica, especificamente nos campos da antropologia e sociologia, destacamos o trabalho de Debert (1999) que analisa as tendências nas abordagens sobre o envelhecimento em décadas recentes.

De natureza mais descritiva, duas outras publicações devem ser mencionadas: o Catálogo de Teses e Dissertações na Área do Envelhecimento (Prado et al., 1999) e o Catálogo de Cursos de Especialização, Mestrado em Geriatria, Gerontologia e Áreas Afins (Prado e Lima, 2000). Esses trabalhos informam, a partir de análises preliminares desenvolvidas sobre bases de dados constituídas no Centro de Referência e Documentação sobre Envelhecimento (CRDE, 2002), da Universidade Aberta da Terceira Idade (UnATI, 2002) da Universidade do Estado do Rio de Janeiro (Uerj), a respeito de algumas características fundamentais desse campo. Em primeiro lugar, a produção de dissertações e teses relativas ao processo de envelhecimento humano vem crescendo de forma exponencial a partir da década de 1970: 7\% dos trabalhos foram concluídos entre os anos de 1975 e 1979; uma terça parte se situa nos anos 80 ; os mais de $60 \%$ restantes foram finalizados entre $1990 \mathrm{e}$ 1998 (Prado et al., 1999); essas obras são originárias de várias instituições, não apenas as que contam com programas de pós-graduação em Gerontologia; e, as dissertações e teses e os programas de pós-graduação encontram-se relacionados com um amplo leque de áreas do conhecimento (Prado e Lima, 2000).

É de se imaginar que a produção científica nacional referente ao processo de envelhecimento humano apresente perfil semelhante. $\mathrm{O}$ nosso estudo acerca dos grupos de pesquisa que operam no Brasil busca contribuir para o conhecimento das características dessa produção nesse cenário nacional de crescente interesse pelo processo de envelhecimento humano.

\section{Procedimentos metodológicos}

Um estudo, em particular, correspondeu ao norte para nossa iniciativa. Guimarães et al. publicaram, em 2001, artigo analisando a capacidade instalada da pesquisa em epidemiologia no Brasil, sua distribuição geográfica e institucional, os pesquisadores e os estudantes, os temas das pesquisas, os padrões de divulgação do conhecimento gerado no interior dos grupos de pesquisa que operam nas instituições em to- 
do o País. Os dados utilizados nesse estudo foram organizados a partir do Diretório dos Grupos de Pesquisa no Brasil, em sua versão 4.0, desenvolvido pelo Conselho Nacional de Desenvolvimento Científico e Tecnológico (CN$\mathrm{Pq}, 2003)$. O critério para o reconhecimento dos grupos que compõem a massa crítica em epidemiologia foi o desenvolvimento, pelo menos, de uma linha de pesquisa nessa subárea, conforme definido pelo pesquisador líder de cada grupo.

O Diretório dos Grupos de Pesquisa corresponde a projeto desenvolvido desde 1992, tendo por objetivo atingir a totalidade do universo da pesquisa nacional; constam do Diretório pouco mais de $80 \%$ dos grupos em atividade. A última versão disponível na Internet (4.1), compreende 11.760 grupos, 49.956 pesquisadores de 224 instituições brasileiras, conforme divulgado na página eletrônica do CNPq. Atualmente encontra-se em andamento a coleta de dados correspondente ao Censo 2002 do Diretório (CNPq, 2003a).

Tomamos a versão 4.1 do Diretório do Grupo de Pesquisa do CNPq. Não nos foi possível, porém, identificar os grupos de pesquisa que mantêm linhas de pesquisa relativas ao envelhecimento humano a partir da área do conhecimento atribuída pelo pesquisador líder a cada linha de pesquisa, como procederam Guimarães et al. A árvore do conhecimento, tal como apresentada pelo $\mathrm{CNPq}$, não contempla, em qualquer nível, possibilidade de associação de qualquer área à Gerontologia, à Geriatria ou ao envelhecimento.

Cabe registrar que a Coordenação de Aperfeiçoamento de Pessoal de nível Superior (Capes, 2002) inclui nas áreas de conhecimento um item denominado outros, o que se deve, certamente, à necessidade concreta de alocar de alguma forma os programas de pós-graduação existentes que não se enquadram em nenhuma das áreas estabelecidas; assim, os cursos de mestrado e doutorado em Gerontologia e Gerontologia Biomédica em atividade no Brasil estão situados na grande área Outros, Área Multidisciplinar. Esta não é uma exclusividade da Gerontologia, uma vez que há dezenas de outros programas enquadrados de forma semelhante.

Uma vez descartada a via metodológica de caminhar a partir das grandes áreas e suas subdivisões, desenhamos uma alternativa. Como é possível realizar buscas no Diretório a partir de palavras-chave, passamos a utilizar expressões freqüentemente utilizadas na literatura científica em associação ao envelhecimento humano e pesquisamos nos seguintes campos do Diretório: Nome do grupo, Nome da linha de pesquisa e Palavras-chave da linha de pesquisa. Primeiramente, foram utilizadas as seguintes palavras-chave: $i d o s o(a)(s)$ ou velho(a)(s) ou velhice ou envelhecimento ou geriatria ou gerontologia ou aposentadoria. Em seguida foi utilizada a expressão terceira idade.

Esse procedimento, realizado em julho de 2001, possibilitou a identificação de 161 grupos de pesquisa. A leitura crítica desse resultado (incluindo os campos Nome do grupo, Nome da linha de pesquisa, Repercussões do grupo e Produção científica) permitiu sua depuração qualitativa. As palavras-chave utilizadas conduziram a alguns "falso-positivos", ou seja, grupos que não fazem parte do campo de interesse deste estudo, como por exemplo, aqueles referentes a envelhecimento de estruturas metálicas ou de concreto, ou ainda envelhecimento de alimentos, como vinhos ou cachaça; da mesma forma, nomes de localidades como Rio Velho ou outros similares, quando presentes nos campos utilizados na busca, foram separados e eliminados, se voltados para outros temas de estudo. Assim, foram mantidos apenas os trabalhos que, com segurança, fazem parte do universo temático investigado. Finalizamos com 144 grupos de pesquisa, incluindo aqueles que trabalham com procedimentos experimentais utilizando outros seres (ratos, camundongos, drosófilas, insetos, vegetais, leveduras); em todos esses casos, há registros claros realizados pelos líderes desses grupos na base de dados destacando a relevância dos conhecimentos gerados para os pesquisadores que se dedicam ao tema envelhecimento humano. É possível que, por meio dos procedimentos de busca que utilizamos, não tenham sido recuperados grupos que, efetivamente, trabalham com o tema em análise. Um motivo bastante provável estaria relacionado às palavras-chave registradas pelos líderes nos formulários eletrônicos do CNPq. Seria o caso de grupos que se dedicam, por exemplo, ao estudo das doenças crônicas e degenerativas, fortemente ligadas ao processo de envelhecimento: palavras-chave mais específicas desses objetos de pesquisa podem ter sido priorizadas em detrimento daquelas que listamos para o presente trabalho.

Concluímos, então, a primeira etapa, estabelecendo uma base de dados de 144 grupos de pesquisa que têm pelo menos uma linha de 
pesquisa que se refere ao envelhecimento humano. Isto significa que há, neste conjunto, grupos que se dedicam especificamente ao tema em tela, além de grupos que estão centralmente voltados para outras áreas de interesse $\mathrm{e}$ que também mantêm produção de conhecimento relativa ao envelhecimento humano.

A segunda etapa de sistematização das informações levantadas sobre grupos de pesquisa com linhas de pesquisa afeitas ao envelhecimento humano consistiu da identificação desses dois subconjuntos. O primeiro é composto por 43 grupos que denominamos específicos aqueles que têm o envelhecimento humano como tema predominante de estudo, ao qual todas as suas linhas de pesquisa estão associadas. O segundo subconjunto, que nomeamos não específicos, perfaz 101 grupos e corresponde àqueles que têm pelo menos uma linha de pesquisa sobre envelhecimento humano, mas cujo objeto central de estudo é outro.

Aos 144 grupos de pesquisa correspondem 610 linhas de pesquisa. Para os 43 grupos específicos há 92 linhas de pesquisa; a estas é necessário agregar mais 117 linhas que são aquelas enunciadas nos grupos não específicos e que trazem registradas as palavras-chave utilizadas na busca. São, portanto, 209 as linhas de pesquisa descritas pelos líderes como ativas na geração de conhecimento relativo ao envelhecimento humano no Brasil.

Embora utilizando metodologia distinta, vale registrar que o estudo sobre a pesquisa em epidemiologia no Brasil revelou a existência de 176 grupos e 320 linhas de pesquisa voltados para esta área (Guimarães et al., 2001).

\section{Áreas predominantes}

No total da base de dados do Diretório, a grande área predominante do conhecimento que integra o maior número de grupos de pesquisa corresponde à de Ciências da Saúde, seguida com muita proximidade das Engenharias (inclusive Ciências da Computação) e das Ciências Exatas e da Terra; depois vêm as Ciências Biológicas e Ciências Humanas com valores semelhantes; e, decrescendo de forma mais acentuada em número de grupos, as Ciências Agrárias, Ciências Sociais Aplicadas e Lingüística, Letras e Artes, conforme nos mostra a tabela 1.

Ao preencher os formulários que compõem a coleta de dados do Diretório dos Grupos de Pesquisa, o líder indica, a seu próprio critério, uma grande área predominante de atuação $\mathrm{e}$ uma área predominante dentro desta definindo assim a posição do grupo na árvore do conhecimento. Identificamos a presença de grupos com linhas de pesquisa relativas ao processo de envelhecimento humano em sete das oito grandes áreas; conforme nos mostra a tabela 2 . Apenas nas ciências agrárias não houve recuperação de grupos na busca que efetivamos.

É evidente a predominância das ciências da saúde entre os grupos que se voltam para o estudo do envelhecimento humano, inclusive, muito mais acentuada que no conjunto do $\mathrm{Di}$ retório. Para os grupos específicos esta característica é ainda mais marcante. As ciências biológicas desempenham papel importante nesse contexto pois, em conjunto com a saúde, correspondem a cerca de $80 \%$ dos grupos específicos.

\begin{tabular}{lcc}
\hline $\begin{array}{l}\text { Tabela 1 } \\
\text { Distribuição dos grupos de pesquisa segundo as grandes áreas do conhecimento predominantes. }\end{array}$ \\
\hline Grandes áreas predominantes & $\mathbf{n}$ & Grupos de pesquisa \\
& 1.832 & $\mathbf{\%}$ \\
\hline Ciências da saúde & 1.826 & 15,6 \\
Engenharias e ciências da computação & 1.812 & 15,5 \\
Ciências exatas e da terra & 1.720 & 15,4 \\
Ciências biológicas & 1.711 & 14,6 \\
Ciências humanas & 1.352 & 14,5 \\
Ciências agrárias & 930 & 7,5 \\
Ciências sociais aplicadas & 577 & 4,9 \\
Lingüística, letras e artes & $\mathbf{1 1 . 7 6 0}$ & $\mathbf{1 0 0 , 0}$ \\
Total & &
\end{tabular}

Fonte: $\mathrm{CNPq}$ - Diretório dos Grupos de Pesquisa no Brasil, versão 4.1, 2000 
Tabela 2

Distribuição dos grupos que contam com linhas de pesquisa referente ao envelhecimento humano, segundo grandes áreas predominantes. Brasil, 2000.

\begin{tabular}{|c|c|c|c|c|c|c|}
\hline \multirow[t]{3}{*}{$\begin{array}{l}\text { Grandes áreas } \\
\text { predominantes }\end{array}$} & \multicolumn{6}{|c|}{$\begin{array}{l}\text { Grupos com linha de pesquisa } \\
\text { referente ao envelhecimento humano }\end{array}$} \\
\hline & \multicolumn{2}{|c|}{ Específicos } & \multicolumn{2}{|c|}{ Não específicos } & \multicolumn{2}{|c|}{ Total } \\
\hline & $\mathbf{n}$ & $\%$ & $\mathbf{n}$ & $\%$ & $\mathbf{n}$ & $\%$ \\
\hline Ciências da saúde & 26 & 60,5 & 56 & 55,4 & 82 & 56,9 \\
\hline Ciências biológicas & 8 & 18,6 & 17 & 16,8 & 25 & 17,4 \\
\hline Ciências humanas & 4 & 9,3 & 16 & 15,8 & 20 & 13,9 \\
\hline Ciências sociais aplicadas & 4 & 9,3 & 10 & 9,9 & 14 & 9,7 \\
\hline Ciências exatas e da terra & 0 & 0,0 & 1 & 1,0 & 1 & 0,7 \\
\hline Engenharias & 0 & 0,0 & 1 & 1,0 & 1 & 0,7 \\
\hline Lingüística, artes e letras & 1 & 2,3 & 0 & 0,0 & 1 & 0,7 \\
\hline Total & 43 & 100,0 & 101 & 100,0 & 144 & 100,0 \\
\hline
\end{tabular}

Fonte: CNPq - Diretório dos Grupos de Pesquisa no Brasil, versão 4.1, 2000

A tabela 3 nos apresenta detalhamentos da distribuição dos grupos de acordo com a área predominante, mostrando a liderança da saúde coletiva, o que também se dá de forma mais enfática entre os grupos específicos. Embora em proporções distintas, o estudo referente à epidemiologia também apresenta este perfil de destaque para as áreas de saúde coletiva e medicina.

A grande amplitude de áreas do conhecimento a que se vinculam os grupos de pesquisa voltados para o envelhecimento humano corresponde a um achado importante, confirmando as indicações de estudos anteriores que registram teses e dissertações provenientes de programas de pós-graduação inscritos em grande diversidade de áreas e qualificando os registros acerca do amplo leque de abordagens que marca este objeto de estudo (Prado et al., 1999 e Prado e Lima, 2000).

\section{Período de criação dos grupos de pesquisa}

Nas últimas décadas, o Brasil vem experimentando importante desenvolvimento acadêmico; a ampliação dos programas de pós-graduação e a elevação na titulação do corpo docente das universidades têm trazido crescimento no número de grupos de pesquisa e na produção de conhecimento. A própria idéia de formação de grupos de pesquisa pelo CNPq faz parte desse processo, cuja versão 1.0 data de 1993. Mas é necessária alguma cautela na análise dos dados que se seguem, pois o fato de um determinado grupo de pesquisa ter se formado, por exemplo, na década de 1970 não significa que esses pesquisadores iniciaram seus estudos nesse momento; nos setores com maior história e tradição de geração de conhecimento, a pesquisa científica pode ser muito anterior à formação dos grupos. Por outro lado, o fato de um grupo mencionar a existência de uma linha de pesquisa associada ao envelhecimento não significa que a produção de conhecimento sobre esse tema já se tenha iniciado; em vários registros, fica clara a intenção de que essas investigações venham a ser desenvolvidas no futuro. A tabela 4 nos diz sobre o processo de criação dos grupos de pesquisa ao longo das últimas décadas.

De modo geral, o interesse crescente pela temática do envelhecimento humano no Brasil coincide com o processo de formação dos grupos de pesquisa. Os grupos que contam com linhas de pesquisa relativas ao envelhecimento humano apresentam perfil relativo ao ano de sua criação bastante semelhante ao encontrado para o conjunto do Diretório, conforme nos mostra a tabela 5. Entretanto, quando tomamos os grupos específicos, notamos que a parcela dos que foram criados após 1995 é bem mais expressiva. Consideramos que esses dados podem se constituir em elemento de confirmação dos registros que indicam serem recentes e intensas as iniciativas relativas a grande parcela da produção de conhecimento sobre envelhecimento humano em nosso país. 
Tabela 3

Distribuição dos grupos que contam com linhas de pesquisa referente ao envelhecimento humano, segundo área predominante. Brasil, 2000.

\begin{tabular}{|c|c|c|c|c|c|c|}
\hline \multirow[t]{3}{*}{ Áreas predominantes } & \multicolumn{6}{|c|}{$\begin{array}{l}\text { Grupos com linha de pesquisa } \\
\text { referente ao envelhecimento humano }\end{array}$} \\
\hline & \multicolumn{2}{|c|}{ Específicos } & \multicolumn{2}{|c|}{ Não específicos } & \multicolumn{2}{|c|}{ Total } \\
\hline & $\mathbf{n}$ & $\%$ & $\mathbf{n}$ & $\%$ & $\mathbf{n}$ & $\%$ \\
\hline Saúde coletiva & 9 & 20,9 & 13 & 12,9 & 22 & 15,3 \\
\hline Medicina & 5 & 11,6 & 12 & 11,9 & 17 & 11,8 \\
\hline Enfermagem & 6 & 14,0 & 6 & 5,9 & 12 & 8,3 \\
\hline Odontologia & 4 & 9,3 & 7 & 6,9 & 11 & 7,6 \\
\hline Psicologia & 3 & 7,0 & 7 & 6,9 & 10 & 6,9 \\
\hline Educação física & 1 & 2,3 & 7 & 6,9 & 8 & 5,5 \\
\hline Nutrição & 1 & 2,3 & 7 & 6,9 & 8 & 5,5 \\
\hline Serviço social & 3 & 7,0 & 4 & 4,0 & 7 & 4,8 \\
\hline Antropologia & 0 & 0,0 & 5 & 4,9 & 5 & 3,5 \\
\hline Sociologia & 3 & 7,0 & 2 & 2,0 & 5 & 3,5 \\
\hline Genética & 1 & 2,3 & 4 & 4,0 & 5 & 3,5 \\
\hline Educação & 2 & 4,6 & 2 & 2,0 & 4 & 2,8 \\
\hline Morfologia & 1 & 2,3 & 3 & 3,0 & 4 & 2,8 \\
\hline Demografia & 0 & 0,0 & 4 & 4,0 & 4 & 2,8 \\
\hline Biologia geral & 1 & 2,3 & 2 & 2,0 & 3 & 2,0 \\
\hline Bioquímica & 1 & 2,3 & 2 & 2,0 & 3 & 2,0 \\
\hline Imunologia & 0 & 0,0 & 3 & 3,0 & 3 & 2,0 \\
\hline Farmácia & 0 & 0,0 & 2 & 2,0 & 2 & 1,4 \\
\hline $\begin{array}{l}\text { Fisioterapia } \\
\text { e terapia ocupacional }\end{array}$ & 0 & 0,0 & 2 & 2,0 & 2 & 1,4 \\
\hline Fisiologia & 0 & 0,0 & 2 & 2,0 & 2 & 1,4 \\
\hline História & 0 & 0,0 & 1 & 1,0 & 1 & 0,7 \\
\hline Direito & 1 & 2,3 & 0 & 0,0 & 1 & 0,7 \\
\hline Economia & 0 & 0,0 & 1 & 1,0 & 1 & 0,7 \\
\hline $\begin{array}{l}\text { Planejamento } \\
\text { urbano e regional }\end{array}$ & 0 & 0,0 & 1 & 1,0 & 1 & 0,7 \\
\hline Física & 0 & 0,0 & 1 & 1,0 & 1 & 0,7 \\
\hline Engenharia civil & 0 & 0,0 & 1 & 1,0 & 1 & 0,7 \\
\hline Lingüística & 1 & 2,3 & 0 & 0,0 & 1 & 0,7 \\
\hline Total & 43 & 100,0 & 101 & 100,0 & 144 & 100,0 \\
\hline
\end{tabular}

Fonte: CNPq - Diretório dos Grupos de Pesquisa no Brasil, versão 4.1, 2000

\section{Tabela 4}

Distribuição dos grupos de pesquisa, segundo o período de sua criação.

\begin{tabular}{lccc}
\hline Ano de criação & $\mathbf{n}$ & $\begin{array}{c}\text { Grupos de pesquisa } \\
\text { \% }\end{array}$ & \% acumulado \\
\hline até 1974 & 330 & 2,8 & 2,8 \\
$1975-1979$ & 374 & 3,2 & 6,0 \\
$1980-1984$ & 613 & 5,2 & 11,2 \\
$1985-1989$ & 1.035 & 8,8 & 20,0 \\
$1990-1994$ & 2.493 & 21,2 & 41,2 \\
$1995-1999$ & 4.503 & 38,3 & 79,5 \\
2000 & 2.412 & 20,5 & 100,0 \\
Total & $\mathbf{1 1 . 7 6 0}$ & $\mathbf{1 0 0 , 0}$ & \\
\hline
\end{tabular}

Fonte: $\mathrm{CNPq}$ - Diretório dos Grupos de Pesquisa no Brasil, versão 4.1, 2000 
Tabela 5

Distribuição dos grupos que contam com linhas de pesquisa relativas ao envelhecimento humano, segundo período de sua criação. Brasil, 2000.

\begin{tabular}{lcccccr}
\hline Ano de criação & \multicolumn{5}{c}{$\begin{array}{c}\text { Grupos com linha de pesquisa } \\
\text { referente ao envelhecimento humano }\end{array}$} \\
& \multicolumn{2}{c}{ Específicos } & \multicolumn{2}{c}{ Não específicos } & Total \\
& $\mathbf{n}$ & $\mathbf{0}$ & $\mathbf{n}$ & $\mathbf{\%}$ & $\mathbf{n}$ & $\%$ \\
\hline até 1974 & 1 & 2,3 & 5 & 5,0 & 6 & 4,2 \\
$1975-1979$ & 0 & 0,0 & 3 & 3,0 & 3 & 2,1 \\
$1980-1984$ & 1 & 2,3 & 5 & 5,0 & 6 & 4,2 \\
$1985-1989$ & 2 & 4,7 & 16 & 15,8 & 18 & 12,5 \\
$1990-1994$ & 6 & 14,0 & 15 & 14,8 & 21 & 14,6 \\
$1995-1999$ & 21 & 48,8 & 39 & 38,6 & 60 & 41,7 \\
2000 & 12 & 27,9 & 18 & 17,8 & 30 & 20,8 \\
Total & $\mathbf{4 3}$ & $\mathbf{1 0 0 , 0}$ & $\mathbf{1 0 1}$ & $\mathbf{1 0 0 , 0}$ & $\mathbf{1 4 4}$ & $\mathbf{1 0 0 , 0}$ \\
\hline
\end{tabular}

Fonte: CNPq - Diretório dos Grupos de Pesquisa no Brasil, versão 4.1, 2000

Apenas um dos grupos não específicos foi criado em fins da década de 1960 . Os anos 70 trazem o surgimento de mais 8 grupos, dos quais apenas um se encontra especificamente voltado para a temática da saúde do idoso nos aspectos bio-psicossociais, cujo nome é NespiNúcleo de Estudos e Pesquisas do Idoso, na área de enfermagem e instalado na Universidade Federal da Bahia (UFBA). Na década de 1980 é três vezes maior o número de grupos que contam com linha de pesquisa relativa ao envelhecimento humano: 24 grupos, dos quais três foram classificados como específicos: um da área de enfermagem da Universidade Federal de Santa Catarina (UFSC) - trata-se do Núcleo de Estudos da Terceira Idade (Neti), que consta como o primeiro programa universitário de atendimento ao idoso e de formação de recursos humanos no campo gerontológico $(\mathrm{Ca}-$ chioni, 1999); outro também da área de enfermagem da Universidade de São Paulo (USP) de Ribeirão Preto; finalmente, o grupo da área de serviço social da Pontifícia Universidade Católica de São Paulo, cuja repercussão de maior peso corresponde à criação do Programa de Mestrado em Gerontologia no Brasil, que iniciou suas atividades em 1997 e foi credenciado pela Capes em 1999.

A partir de 1990 até o ano 2000, inclusive, foram criados 111 grupos com ao menos uma linha de pesquisa dentro do escopo do presente estudo, o que corresponde a $77,1 \%$ do total estudado; entre estes, 39 são grupos específicos representando $90,7 \%$ dos grupos que se dedicam de forma central ao estudo de aspectos relativos ao envelhecimento humano. Vale destacar que, em 1997, iniciaram-se as atividades do Programa de Pós-Graduação em Gerontologia (mestrado) na Faculdade de Educação da Unicamp e, em 2000, o curso de mestrado no Instituto de Geriatria e Gerontologia da PUC-RS e doutorado em 2001, todos a partir de grupos de pesquisa.

Colocando o foco sobre os grupos especificos, com apenas um formado na década de 1970 , poucos nos anos 80 e uma explosão a partir de 1990, encontramos que esse perfil guarda coerência com a perspectiva de Groisman (1999) que identifica três momentos históricos de destaque para a construção de uma história da velhice no Brasil, a saber: a virada do século 19 para o século 20 , quando uma série de mudanças - referentes aos asilos e instituição das aposentadorias - traria a velhice como uma etapa específica da vida; as décadas de 19601970, com o surgimento das sociedades científicas da área e a constituição de um discurso específico que começa, gradativamente, a ganhar espaços institucionais; finalmente, os anos 90 , quando o envelhecimento, como questão social, alcança proporções até então não registradas, com destaque para os meios de comunicação.

Cachioni (1999), estudando a formação das universidades da terceira idade no Brasil, destaca o pioneirismo do Sesc, na década de 1960, e registra que A partir da década de 1980, podemos verificar que as universidades começam a 
abrir um espaço educacional, tanto para a população idosa como para profissionais interessados no estudo das questões do envelhecimento. Entre os adultos maduros e idosos predomina a oferta de programas de ensino, saúde e lazer, por meio dos quais acredita-se que essa população vem encontrando possibilidades de combinar desenvolvimento de sociabilidade e educação permanente.

Encontramos, com freqüência, nos registros realizados pelos líderes, informações que associam a criação de grupos a partir de atividades assistenciais e/ou extensionistas em suas universidades. Assim, tomando as considerações de Groisman, de Cachioni e as informações dos líderes, consideramos que esse conjunto sugere um papel importante das universidades da terceira idade no surgimento dos grupos de pesquisa, particularmente, os específicos.

A juventude das iniciativas de formação dos grupos de pesquisa especificamente voltados para o envelhecimento humano, a existência de apenas três programas de pós-graduação e o fato de serem todos muito recentes são elementos importantes que reforçam as perspectivas de que a geração de conhecimento sobre envelhecimento humano no Brasil é algo novo.

\section{Distribuição geográfica}

Os dados apresentados na tabela 6 mostram que, acompanhando o padrão de concentração da produção científica brasileira, a região $\mathrm{Su}$ deste conta com $59,7 \%$ dos grupos de pesquisa no campo do envelhecimento humano; valor dessa ordem foi encontrado no estudo acerca da pesquisa em epidemiologia $(60,8 \%)$ e que é semelhante ao presente no total do Diretório (57\%). Na região Sul encontram-se instalados $21,5 \%$ dos grupos que contam com linhas de pesquisa sobre envelhecimento, acompanhando o perfil geral da base de dados do $\mathrm{CNPq}$ (20\%), mas distanciando-se do padrão registrado na pesquisa em epidemiologia $(9,7 \%)$. A região Nordeste abriga 13,9\% dos grupos que produzem conhecimento sobre envelhecimento, guardando similaridade com o encontrado no Diretório (15\%) e diferindo do resultado referente à pesquisa em epidemiologia (21,6\%). A região Centro-Oeste participa com $4,9 \%$ dos grupos que mantêm investigações sobre envelhecimento humano, não havendo registro de grupos na região Norte (Guimarães et al., 2001).
A tabela 7 apresenta o detalhamento por unidade da federação mostrando-nos que nos estados de São Paulo, Rio Grande do Sul e Rio de Janeiro encontram-se 71,6\% dos grupos que contam com pelo menos uma linha de pesquisa sobre envelhecimento humano e que em São Paulo e Rio Grande do Sul estão 69,8\% dos grupos específicos. Esse perfil corresponde à especificidade do campo em questão e pode ser compreendido, em parte, pelo fato de que é nos dois estados que se encontram os três programas de pós-graduação em gerontologia do Brasil. Também é necessário considerar nomes de destaque na pesquisa sobre velhos, velhice e envelhecimento que se encontram no interior de universidades situadas no eixo Rio-São Paulo, porém atuando em outros programas de pós-graduação, situados em áreas como epidemiologia, medicina, e antropologia, por exemplo. Tomando por referência os dados registrados no total da base do Diretório do $\mathrm{CNPq}$, encontramos relativa similaridade ao quadro nacional: São Paulo (31,0\%), Rio de Janeiro (16,3\%), Rio Grande do Sul (10,2\%) abrigam mais da metade dos grupos de pesquisa. Quanto à epidemiologia, os grupos encontram-se mais concentrados nos estados de São Paulo (31,3\%) e Rio de Janeiro (21,6\%), com relativa participação da Bahia, Minas Gerais, Pernambuco e Rio Grande do Sul, observando que os programas de pósgraduação stricto sensu que comportam o campo epidemiológico estão presentes em maior número de instituições e de unidades da federação que os dedicados à gerontologia.

\section{Distribuição institucional}

Esse levantamento nos revela que 47 das instituições de ensino superior mantêm grupos de pesquisa que desenvolvem pelo menos uma linha de pesquisa relativa ao envelhecimento humano. Entre estas, a Universidade de São Paulo (USP), a Pontifícia Universidade Católica do Rio Grande do Sul (PUC-RS) e a Universidade Estadual de Campinas (Unicamp) aparecem como líderes, indicadas pelo número de grupos ativos que mantêm. A Universidade Federal do Rio Grande do Sul (UFGRS), a Universidade Federal de Minas Gerais (UFMG) e a Universidade do Estado do Rio de Janeiro (Uerj) apresentam peso relativamente importante no conjunto, conforme mostra a tabela 8 .

Vale destacar que no Rio Grande do Sul um estudo multidimensional acerca dos idosos 
Tabela 6

Distribuição dos grupos que contam com linhas de pesquisa referente ao envelhecimento humano, segundo região geográfica. Brasil, 2000.

\begin{tabular}{lcccccr}
\hline Região geográfica & \multicolumn{5}{c}{$\begin{array}{c}\text { Grupos com linha de pesquisa } \\
\text { referente ao envelhecimento humano }\end{array}$} \\
& \multicolumn{2}{c}{ Específicos } & \multicolumn{2}{c}{ Não específicos } & Total \\
& $\mathbf{n}$ & $\mathbf{\%}$ & $\mathbf{n}$ & $\mathbf{\%}$ & $\mathbf{n}$ & $\mathbf{\%}$ \\
\hline Sudeste & 23 & 53,5 & 63 & 62,4 & 86 & 59,7 \\
Sul & 13 & 30,2 & 18 & 17,8 & 31 & 21,5 \\
Nordeste & 6 & 14,0 & 14 & 13,9 & 20 & 13,9 \\
Centro-Oeste & 1 & 2,3 & 6 & 5,9 & 7 & 4,9 \\
Norte & 0 & 0,0 & 0 & 0,0 & 0 & 0,0 \\
Total & $\mathbf{4 3}$ & $\mathbf{1 0 0 , 0}$ & $\mathbf{1 0 1}$ & $\mathbf{1 0 0 , 0}$ & $\mathbf{1 4 4}$ & $\mathbf{1 0 0 , 0}$ \\
\hline
\end{tabular}

Fonte: $\mathrm{CNPq}$ - Diretório dos Grupos de Pesquisa no Brasil, versão 4.1, 2000

Tabela 7

Distribuição dos grupos que contam com linhas de pesquisa referente ao envelhecimento humano, segundo Estado. Brasil, 2000.

\begin{tabular}{|c|c|c|c|c|c|c|}
\hline \multirow[t]{3}{*}{ Unidade da Federação } & \multicolumn{6}{|c|}{$\begin{array}{l}\text { Grupos com linha de pesquisa } \\
\text { referente ao envelhecimento humano }\end{array}$} \\
\hline & \multicolumn{2}{|c|}{ Específicos } & \multicolumn{2}{|c|}{ Não específicos } & \multicolumn{2}{|c|}{ Total } \\
\hline & $\mathbf{n}$ & $\%$ & $\mathbf{n}$ & $\%$ & $\mathbf{n}$ & $\%$ \\
\hline São Paulo & 18 & 41,9 & 38 & 37,6 & 56 & 38,9 \\
\hline Rio Grande do Sul & 12 & 27,9 & 14 & 13,9 & 26 & 18,1 \\
\hline Rio de Janeiro & 3 & 7,0 & 18 & 17,8 & 21 & 14,6 \\
\hline Minas Gerais & 1 & 2,3 & 7 & 6,9 & 8 & 5,6 \\
\hline Bahia & 2 & 4,7 & 5 & 5,0 & 7 & 4,9 \\
\hline Distrito Federal & 1 & 2,3 & 4 & 3,7 & 5 & 3,5 \\
\hline Pernambuco & 3 & 7,0 & 2 & 2,0 & 5 & 3,5 \\
\hline Paraíba & 1 & 2,3 & 3 & 3,0 & 4 & 2,8 \\
\hline Santa Catarina & 1 & 2,3 & 2 & 2,0 & 3 & 2,1 \\
\hline Ceará & 0 & 0,0 & 2 & 2,0 & 2 & 1,4 \\
\hline Goiás & 0 & 0,0 & 2 & 2,0 & 2 & 1,4 \\
\hline Paraná & 0 & 0,0 & 2 & 2,0 & 2 & 1,4 \\
\hline Espírito Santo & 1 & 2,3 & 0 & 0,0 & 1 & 0,7 \\
\hline Rio Grande do Norte & 0 & 0,0 & 1 & 1,0 & 1 & 0,7 \\
\hline Sergipe & 0 & 0,0 & 1 & 1,0 & 1 & 0,7 \\
\hline Total & 43 & 100,0 & 101 & 100,0 & 144 & 100,0 \\
\hline
\end{tabular}

Fonte: $\mathrm{CNPq}$ - Diretório dos Grupos de Pesquisa no Brasil, versão 4.1, 2000

gaúchos envolvendo várias instituições de ensino superior foi mencionado por vários grupos de pesquisa. A experiência parece ter sido um elemento de contribuição para a formação de grupos que referem linhas de pesquisa sobre envelhecimento em muitas universidades nesse Estado.

Em linhas gerais, a pesquisa epidemiológica acontece aproximadamente nas mesmas ins- tituições em que se encontram os grupos que contam com linhas de pesquisa sobre envelhecimento humano.

Confirmando registros anteriores, embora mais concentrada em algumas universidades das regiões Sudeste e Sul, a pesquisa sobre envelhecimento se encontra, ao mesmo tempo, pulverizada em instituições de ensino superior por quase todo o País (Prado e Lima, 2000). 
Tabela 8

Distribuição dos grupos que contam com linhas de pesquisa referentes ao envelhecimento humano, segundo instituição. Brasil, 2000.

\begin{tabular}{|c|c|c|c|c|c|c|}
\hline \multirow[t]{3}{*}{ Instituição } & \multicolumn{6}{|c|}{$\begin{array}{l}\text { Grupos com linha de pesquisa } \\
\text { referente ao envelhecimento humano }\end{array}$} \\
\hline & \multicolumn{2}{|c|}{ Específicos } & \multicolumn{2}{|c|}{ Não específicos } & \multicolumn{2}{|c|}{ Total } \\
\hline & $\mathbf{n}$ & $\%$ & $\mathbf{n}$ & $\%$ & $\mathbf{n}$ & $\%$ \\
\hline Universidade de São Paulo & 5 & 11,6 & 10 & 9,9 & 15 & 10,4 \\
\hline Pontifícia Universidade Católica do Rio Grande do Sul & 7 & 16,3 & 5 & 4,9 & 12 & 8,3 \\
\hline Universidade Estadual de Campinas & 4 & 9,3 & 8 & 7,9 & 12 & 8,3 \\
\hline Universidade do Estado do Rio de Janeiro & 2 & 4,6 & 5 & 4,9 & 7 & 4,9 \\
\hline Universidade Federal de Minas Gerais & 0 & 0,0 & 7 & 6,9 & 7 & 4,9 \\
\hline Universidade Federal do Rio Grande do Sul & 2 & 4,6 & 5 & 4,9 & 7 & 4,9 \\
\hline Universidade Estadual Paulista Júlio de Mesquita Filho & 2 & 4,6 & 5 & 4,9 & 7 & 4,9 \\
\hline Universidade Federal da Bahia & 1 & 2,3 & 4 & 4,0 & 5 & 3,5 \\
\hline Universidade Federal Fluminense & 0 & 0,0 & 5 & 4,9 & 5 & 3,5 \\
\hline Universidade Federal de São Paulo & 0 & 0,0 & 5 & 4,9 & 5 & 3,5 \\
\hline Universidade Federal de Pernambuco & 2 & 4,6 & 2 & 2,0 & 4 & 2,8 \\
\hline Universidade de Brasília & 0 & 0,0 & 4 & 4,0 & 4 & 2,8 \\
\hline Fundação Oswaldo Cruz & 0 & 0,0 & 3 & 3,0 & 3 & 2,1 \\
\hline Universidade Federal da Paraíba & 1 & 2,3 & 2 & 2,0 & 3 & 2,1 \\
\hline Universidade Federal do Rio de Janeiro & 0 & 0,0 & 3 & 3,0 & 3 & 2,1 \\
\hline Universidade Federal de São Carlos & 1 & 2,3 & 2 & 2,0 & 3 & 2,1 \\
\hline Universidade Camilo Castelo Branco & 1 & 2,3 & 2 & 2,0 & 3 & 2,1 \\
\hline Universidade de Taubaté & 2 & 4,6 & 1 & 1,0 & 3 & 2,1 \\
\hline Faculdade de Ciências Médicas da Sta. Casa de São Paulo & 1 & 2,3 & 1 & 1,0 & 2 & 1,4 \\
\hline Pontifícia Universidade Católica de São Paulo & 2 & 4,6 & 0 & 0,0 & 2 & 1,4 \\
\hline Universidade Católica de Brasília & 1 & 2,3 & 1 & 1,0 & 2 & 1,4 \\
\hline Universidade Estadual de Santa Cruz & 1 & 2,3 & 1 & 1,0 & 2 & 1,4 \\
\hline Universidade Federal do Ceará & 0 & 0,0 & 2 & 2,0 & 2 & 1,4 \\
\hline Universidade Metodista de Piracicaba & 0 & 0,0 & 2 & 2,0 & 2 & 1,4 \\
\hline Universidade de Passo Fundo & 1 & 2,3 & 1 & 1,0 & 2 & 1,4 \\
\hline Faculdade de Medicina de São José do Rio Preto & 0 & 0,0 & 1 & 1,0 & 1 & 0,7 \\
\hline Pontifícia Universidade Católica de Campinas & 0 & 0,0 & 1 & 1,0 & 1 & 0,7 \\
\hline Pontifícia Universidade Católica do Paraná & 0 & 0,0 & 1 & 1,0 & 1 & 0,7 \\
\hline Pontifícia Universidade Católica do Rio de Janeiro & 0 & 0,0 & 1 & 1,0 & 1 & 0,7 \\
\hline Universidade Católica de Goiás & 0 & 0,0 & 1 & 1,0 & 1 & 0,7 \\
\hline Universidade de Caxias do Sul & 1 & 2,3 & 0 & 0,0 & 1 & 0,7 \\
\hline Universidade Estadual de Maringá & 0 & 0,0 & 1 & 1,0 & 1 & 0,7 \\
\hline Universidade Estadual da Paraíba & 0 & 0,0 & 1 & 1,0 & 1 & 0,7 \\
\hline Universidade Federal do Espírito Santo & 1 & 2,3 & 0 & 0,0 & 1 & 0,7 \\
\hline Universidade Federal de Goiás & 0 & 0,0 & 1 & 1,0 & 1 & 0,7 \\
\hline Universidade Federal do Rio Grande do Norte & 0 & 0,0 & 1 & 1,0 & 1 & 0,7 \\
\hline Universidade Federal de Santa Catarina & 1 & 2,3 & 0 & 0,0 & 1 & 0,7 \\
\hline Universidade Federal do Sergipe & 0 & 0,0 & 1 & 1,0 & 1 & 0,7 \\
\hline Universidade Federal de Uberlândia & 1 & 2,3 & 0 & 0,0 & 1 & 0,7 \\
\hline Universidade Metodista de São Paulo & 0 & 0,0 & 1 & 1,0 & 1 & 0,7 \\
\hline Universidade de Ribeirão Preto & 0 & 0,0 & 1 & 1,0 & 1 & 0,7 \\
\hline Universidade de Cruz Alta & 0 & 0,0 & 1 & 1,0 & 1 & 0,7 \\
\hline Universidade Regional do Noroeste & 0 & 0,0 & 1 & 1,0 & 1 & 0,7 \\
\hline do Estado do Rio Grande do Sul & & & & & & \\
\hline Universidade de Santa Cruz do Sul & 1 & 2,3 & 0 & 0,0 & 1 & 0,7 \\
\hline Universidade do Vale do Rio dos Sinos & 0 & 0,0 & 1 & 1,0 & 1 & 0,7 \\
\hline Universidade Salgado de Oliveira & 1 & 2,3 & 0 & 0,0 & 1 & 0,7 \\
\hline Universidade de Pernambuco & 1 & 2,3 & 0 & 0,0 & 1 & 0,7 \\
\hline Total & 43 & 100,0 & 101 & 100,0 & 144 & 100,0 \\
\hline
\end{tabular}

Fonte: $\mathrm{CNPq}$ - Diretório dos Grupos de Pesquisa no Brasil, versão 4.1, 2000 


\section{Considerações finais}

A pesquisa relativa à velhice, ao velho e ao processo de envelhecimento acontece, predominantemente, na área das ciências da saúde, com importante participação das ciências biológicas. As ciências humanas e as sociais aplicadas também desempenham papel importante nas pesquisas relativas à temática. De fato, o envelhecimento humano interessa a pesquisadores de muitos campos da produção de conhecimento.

Os grupos de pesquisa ativos nessa produção de conhecimento estão concentrados nas regiões Sudeste e Sul, mais especificamente nos estados de São Paulo, Rio Grande do Sul e Rio de Janeiro, desenvolvendo suas investigações, principalmente, na USP, PUC-RS e Unicamp; em algum grau, esta característica está associada aos programas de pós-graduação instalados na PUC-RS, na Unicamp e na Pontifícia Universidade Católica de São Paulo (PUC-SP). Simultaneamente à concentração, a pesquisa sobre envelhecimento humano está pulverizada em várias unidades da federação e muitas instituições de ensino superior.

Não se trata, porém, de um conjunto uniforme. Há aqueles grupos que tomam o envelhecimento como uma parte de alguma área de interesse anteriormente definida, esta sim, central em seus trabalhos. Pioneiros na geração de conhecimento sobre envelhecimento correspondem a $70 \%$ dos grupos de pesquisa que trabalham com essa temática. Mas para todos esses, o envelhecimento é um tema, tratado no interior de sua área / sub-área previamente estabelecida, seja ela, sociologia, antropologia, demografia, medicina, saúde coletiva, educação física, ou qualquer outra.
E há os grupos que se voltam especificamente para o estudo do processo de envelhecimento humano. No seu conjunto, correspondem a $30 \%$ do universo desta investigação. $O$ olhar das ciências da saúde e das ciências biológicas é aqui muito mais intenso, materializado, principalmente, por meio da saúde coletiva, da medicina, da enfermagem e da odontologia. São grupos criados há bem pouco tempo, particularmente, a partir de 1995. É pequeno o número de grupos vinculados a programas de pós-graduação stricto sensu, mesmo porque estes também se apresentam em número reduzido. O quadro traçado acima corresponde ao perfil geral desses grupos específicos e, evidentemente, não se pode, aqui também, falar em homogeneidade. Há alguns grupos altamente qualificados, com geração de conhecimento reconhecida nacional e/ou internacionalmente, desenvolvendo suas pesquisas em associação com renomados programas de pósgraduação. Como também existem os que dão seus primeiros passos com o raiar do novo milênio; são sanitaristas, enfermeiros, médicos, odontólogos, psicólogos, assistentes sociais, nutricionistas, educadores, entre outros, que se voltam para o processo de envelhecimento, muitas vezes a partir de experiências assistenciais e/ou extensionistas e que mencionam com maior freqüência a geriatria e a gerontologia em seus registros sobre os grupos que compõem. Talvez, neste espaço, estejamos mais próximos dos caminhos que conduzem às iniciativas voltadas para a constituição desta área - a gerontologia -, que parece ainda não ter reunido forças para constar da árvore do conhecimento, em que pese todo o destaque que já conquistou em vários outros campos. 


\section{Referências bibliográficas}

Cachioni M 1999. Universidades da terceira idade: das origens à experiência brasileira, pp. 141-178. In AL Néri e GG Debert (orgs.) Velhice e sociedade. Papirus, Campinas.

Centro de Referência e Documentação sobre Envelhecimento (CRDE) da Universidade Aberta da Terceira Idade (UnATI-UERJ). [citado 15 setembro 2002]. Disponível em <www.unati.uerj.br/crde/crde.htm>

Coordenação de Aperfeiçoamento de Pessoal de Nível Superior (CAPES), [04 Abril 2002]. Disponível em $<$ www.capes.gov.br/>

Conselho Nacional de Desenvolvimento Científico e Tecnológico (CNPq) [5 maio 2003]. Diretório dos Grupos de Pesquisa do Brasil, versão 4.1. Disponível em $<$ www.cnpq.br/plataformalattes/dgp/versao4/>

Conselho Nacional de Desenvolvimento Científico e Tecnológico (CNPq) [5 maio 2003a]. Diretório dos Grupos de Pesquisa do Brasil, Censo 2002. Disponível em $<$ lattes.cnpq.br/censo2002/>

Debert GG 1999. A reinvenção da velhice. Edusp-FAPESP, São Paulo.

Groisman D 1999. Velhice e história: perspectivas teóricas. Cadernos do IPUB 1(10-2a ed.):43-56

Groisman D [citado 24 agosto 2002]. A velhice, entre o normal e o patológico. História Ciência e Saúde. [online]. jan. 2002, 9(1):61-78. Disponível em <www. scielo.br/scielo.php?script=sci_arttext\&pid=S0104$59702002000100004 \& \operatorname{lng}=\mathrm{pt} \& \mathrm{nrm}=\mathrm{iso}>$. ISSN 0104 5970.
Guimarães R, Lourenço R \& Cosac S [citado 24 agosto 2002]. A pesquisa em epidemiologia no Brasil. Revista de Saúde Pública. [online]. ago. 2001, 35(4):321340. Disponível em <www.scielo.br/scielo.php?script $=$ sci_arttext\&pid $=$ S0034-89102001000400001 $\& \operatorname{lng}=\mathrm{pt} \& \mathrm{nrm}=\mathrm{iso}>$. ISSN 0034-8910.

Neri AL \& Debert GG 1999. Apresentação. In AL Néri \& GG Debert (orgs.) Velhice e sociedade. Papirus, Campinas.

Prado SD \& Tavares EL 1999. Produção acadêmica sobre envelhecimento e velhice: alguns registros preliminares acerca da produção da pós-graduação, pp. 7-9. In SD Prado, EL Tavares \& FRC Silva. Catálogo de teses e dissertações na área do envelhecimento humano. CRDE-UnATI-CRDE: Rio de Janeiro.

Prado SD \& Lima MA 2000. Catálogo de cursos de especialização, mestrado e doutorado em geriatria, gerontologia e áreas afins. CRDE-UnATI-Uerj: Rio de Janeiro.

Universidade Aberta da Terceira Idade (UnATI). [citado 15 setembro 2002]. Disponível em <www.unati. uerj.br>

Artigo apresentado em 11/8/2003

Aprovado em 24/8/2003

Versão final apresentada em 11/11/2003 